\title{
Impacto de la Beca Universal en retención escolar y cumplimiento del Ciclo Educativo.
}

\section{Luis Carlos Herrera $\mathbf{M}^{1,2,4 *}$., Virginia Torres-Lista ${ }^{1,2,4}$,, Markelda Montenegro ${ }^{3,4}$., Carmen Forero ${ }^{5}$.}

${ }^{1}$ Investigador de la Universidad Católica Santa María La Antigua (USMA), ${ }^{2}$ Miembro del Sistema Nacional de Investigación (SNI), ${ }^{3}$ Presidenta del Centro de Investigaciones Científicas de Ciencias Sociales (CENICS), ${ }^{4}$ Miembro del Centro de Centro de Investigaciones Científicas de Ciencias Sociales (CENICS), ${ }^{5}$ Profesora de Estadística de la Universidad de Panamá.

*Autores para correspondencia. Email: $\underline{\text { herreram@usma.com.pa }}$

Recibido: 17 de marzo de 2019

Aceptado: 18 de abril de 2019

\section{Resumen}

Han trascurrido más de 8 años que entró en vigor la Ley N. ${ }^{\circ} 40$ del 23 de agosto de 2010, que regula el Programa de Beca Universal y modifica un artículo de la Ley 8 de 2010, sin tener una evaluación de sus resultados, particularmente en el tema de la retención escolar. En este artículo se presenta el análisis de los aspectos teóricos del programa, el impacto en la retención escolar condicionada al cumplimiento del ciclo de estudio, básica, premedia y media, en el tiempo esperado. Por lo tanto, estamos ante otra mirada de problema, más allá de la deserción o el fracaso escolar. La metodología se plantea en dos etapas: la primera consistió en el análisis de toda la normativa del programa de la beca universal desde la sociología jurídica; la segunda fase es cuantitativa, en la cual el tipo de impacto se mide a partir de dos variables, "REDU = Retención y Efectividad Educativa", esta mide a nivel \% la capacidad del sistema de lograr retener a los estudiantes pero condicionada a que finalicen en el tiempo esperado, $\mathrm{y}$ la segunda variable "REZ = esta mide en cifras absolutas la cantidad de estudiantes que están dentro del sistema educativo pero que en determinado ciclo no logran terminarlo en el tiempo esperado. Dentro de los principales resultados; a nivel de la norma se evidencian diversos vacíos que afectaron la efectividad del programa. Por otro lado, en la dimensión cuantitativa se puede demostrar que en el ciclo de básica de un $87 \%$ de efectividad se incrementa a un $94 \%$, en el caso del ciclo de la Premedia y Media, de $23 \%$ se aumenta a un $51 \%$ de efectividad. Estos resultados permiten diseñar la metodología para un sistema de seguimiento y evaluación; que requieren incorporar para la mejora del programa.

Palabras Claves: Sistema educativo, beca universal, retención escolar, sociología jurídica, políticas educativas.

\footnotetext{
Abstract

It has been more than 8 years since Law No. 1 came into force. 40 of August 23, 2010, which regulates the Universal Scholarship Program and modifies an article of Law 8 of 2010, without having an evaluation of its results, particularly on the issue of school retention. This article presents the analysis
} 
Invest. pens. crit. (ISSN 1812-3864)

Vol. 7, No. 1, enero- abril 2019

pp. 05-26

of the theoretical aspects of the program, the impact on school retention conditioned to the completion of the study cycle, basic, premedia and media, in the expected time. Therefore, we are facing another problem look, beyond school dropout or failure. The methodology is presented in two stages: the first consisted in the analysis of all the regulations of the universal scholarship program from the legal sociology; the second phase is quantitative, in which the type of impact is measured from two variables, "REDU = Retention and Educational Effectiveness", which measures at \% level the capacity of the system to retain students but conditioned to the completion in the expected time, and the second variable "REZ = this measures in absolute numbers the number of students that are within the educational system but in a certain cycle do not manage to finish it in the expected time. Within the main results; At the level of the norm, there are several gaps that affected the effectiveness of the program. On the other hand, in the quantitative dimension it can be shown that in the basic cycle of $87 \%$ effectiveness increases to $94 \%$, in the case of the Premedia and Media cycle, 23\% is increased to $51 \%$ of effectiveness. These results allow designing the methodology for a monitoring and evaluation system; that they need to incorporate for the improvement of the program.

Key words: Educational system, universal scholarship, school retention, legal sociology, educational policies.

\section{Introducción}

No existen investigaciones que arrojen los resultados del Programa de Beca Universal, creado por la Ley 40 de 2010, el cual fue modificado por la Ley No.14, de 12 de agosto de 2014 y modificaciones posteriores de la Ley 389 de 2017, en cuanto a la retención escolar en el cumplimiento del todo el ciclo escolar; y conocer si la niñez está terminando su escuela en el tiempo. Algo está ocurriendo y no lo estamos analizando, ¿Cuál es la situación de la retención escolar en la Básica, Premedia y Media?

Entre los objetivos del programa de Beca Universal, se encuentran prevenir la deserción escolar en estudiantes con problemas socioeconómicos, y aumentar la retención en las escuelas de aquellos estudiantes de básica, premedia y media, a través de un apoyo económico, que les permita atender sus necesidades escolares básicas, siempre que mantengan un promedio mínimo de 3.0.

Sin embargo, no hay claridad de cuál es el concepto de beca. En las reglamentaciones del IFARHU, específicamente en el "Manual de Procedimientos de Beca Universal" aprobado en el 2016, se encuentran dos acepciones, por un lado, la definición de la beca universal, como "apoyo económico" que se brinda a estudiantes de básica, premedia y media, hasta la culminación de sus estudios para lograr los objetivos previstos en la Ley", y al mismo tiempo, la definen como "asistencia económica educativa condicionada", cuya finalidad es prevenir y contrarrestar la deserción escolar, es decir que para recibir el pago, los estudiantes deben cumplir los requisitos, pero además expresa de manera taxativa los fines de la beca. En ambas definiciones de este reglamento, nos muestra que es un subsidio educativo, con requisitos mínimos, que recoge los objetivos que señala la Ley 14 de 2014.

Estamos ante una contradicción conceptual; por ejemplo: la palabra Beca, según el diccionario de la Lengua Española, es un término que se refiere a "un aporte económico que se concede a aquellos estudiantes o investigadores con el fin de llevar a cabo estudios o investigaciones". Aunque pueden ser de distintos tipos. Se les otorga a las personas que no pueden costear el valor del estudio o investigación. Es una forma de darle oportunidades a los estudiantes que no tienen recurso para 
Invest. pens. crit. (ISSN 1812-3864)

Vol. 7, No. 1, enero- abril 2019

pp. $05-26$

financiar sus estudios, pero que tienen capacidades y méritos y sin este apoyo se perdería estos talentos. (RAE, 2014)

Por otro lado, la alusión "universal", hace referencia a que su beneficio se extiende a todos los integrantes de un grupo, que tienen alcance sin distinción; es decir, es un beneficio previsto para favorecer a todos los estudiantes de básica, premedia y media, aunque en principio, no se implementó en los centros educativos particulares.

El programa establece que el estudiante debe tener una calificación mínima de 3.0 del promedio por bimestre y aunque en las distintas modificaciones adiciona la participación de los padres, buena conducta de los estudiantes, entre otras, es sin duda una política educativa que debió tener un diagnóstico y línea base que permitiera tener indicadores para analizar su impacto. Mantener los mismos criterios de rendimiento, puede generar desinterés de lograr mejores resultados de aprendizaje, porque van a recibir el beneficio económico, sin tener que cumplir más exigencias académicas.

Por lo tanto, el principal cuestionamiento es el nombre del programa, ¿es realmente una beca? Recordando que el objetivo del programa es prevenir la deserción escolar, ¿Se han logrado los objetivos del programa? Tomando en cuenta que, aunque la beca dice "universal", en el caso de escuelas particulares, sólo se benefician a los estudiantes que en la matrícula y mensualidad anual no superan la suma de dos mil balboas, (B/2,000.00), a diferencia de las escuelas y colegios oficiales que no tiene ninguna limitante.

El programa de beca universal genera diversas opiniones, por ejemplo; dirigentes magisteriales señalan que no es una beca, y tampoco disminuirá el índice de deserción escolar y fracasos, y que premiar a estudiantes puede provocar insatisfacción en los que sí se esfuerzan por sacar buenas calificaciones, y antes para ganar una beca debías esforzarse y probar eran los mejores en el aula. (Pino, 2012). Sin embargo, lo que debemos tener claro, es que el programa no se le debe medir en aspectos ajenos a sus objetivos.

Dicho esto, la investigación analiza la retención escolar, y su vinculación con los beneficios de la Beca Universal en la educación básica, media y premedia; la permanencia y culminación de los estudios que permita medir el impacto del programa de la beca universal.

\subsection{Consideraciones Generales del Programa Beca Universal.}

Los programas deben ser parte de las políticas públicas educativas, integrales y articuladas a otras acciones sociales del Estado para alcanzar la calidad de la educación; es un tema donde intervienen muchos factores; la calidad de los docentes, los contenidos, la pedagogía, el ambiente escolar, la participación de padres, madres y acudientes en el proceso de enseñanza aprendizaje, el uso de las tecnologías; así como las distintas ofertas culturales y deportivas para promover los talentos del estudiantado; nuevas metodologías para una educación de excelencia que garantice la formación transformadora, el pensamiento crítico y reflexivo; que potencie su creatividad e imaginación; fortalezca otros procesos básicos y complejos del pensamiento, con conocimientos, liderazgo, valores, habilidades y destrezas para la vida. 
Invest. pens. crit. (ISSN 1812-3864)

Vol. 7, No. 1, enero- abril 2019

pp. $05-26$

Según la memoria del IFARHU (2012), el programa da inicio el 30 de agosto de 2010, y se implementa el plan piloto en la Provincia de Bocas del Toro, donde las autoridades anunciaron que serían beneficiados 83 mil 490 estudiantes. En total se atendieron en ese año, 214,107 mil estudiantes a nivel nacional; y se identifica la beca como un apoyo económico.

En el año 2011 se atendieron a 307, 949 estudiantes, el programa incluyó a estudiantes de básica de cuarto a sexto grado, y de secundaria de primero a sexto año y es hasta el 2013, que se empieza a incluir a las escuelas privadas básicas y secundarias; es decir este Programa se ejecuta directamente en todas las escuelas y colegios oficiales de la república y desde el año 2013 en las escuelas y colegios particulares que cumplan con lo establecido en la Ley. (IFARHU, 2012)

En el 2012, se beneficiaron 481,788 estudiantes, para el 2013 eran 341,397 estudiantes, en el 2014, se beneficiaron 500 mil estudiantes; en el 2015 fueron 554, 953 mil estudiantes en el país, en el 2016 se otorgó beca universal a 607 mil estudiantes de escuelas y colegios oficiales y 40 mil de escuelas particulares y en el 2017 aumento a 648, 442 estudiantes. (IFARHU, 2012)

\section{Cuadro No 1 Total de Beca Universal Concedidas Por El IFARHU} Según Nivel De Estudio: Años 2010 - 2016

\begin{tabular}{|l|r|r|r|r|r|r|r|}
\hline $\begin{array}{c}\text { Nivel de } \\
\text { Estudio }\end{array}$ & \multicolumn{1}{|c|}{$\mathbf{2 0 1 0}$} & $\mathbf{2 0 1 1}$ & $\mathbf{2 0 1 2}$ & $\mathbf{2 0 1 3}$ & $\mathbf{2 0 1 4}$ & $\mathbf{2 0 1 5}$ & $\mathbf{2 0 1 6}$ \\
\hline \multicolumn{1}{|c|}{ Total } & $\mathbf{2 1 4 , 1 0 7}$ & $\mathbf{3 7 8 , 0 3 3}$ & $\mathbf{4 8 1 , 7 8 8}$ & $\mathbf{4 7 8 , 5 7 4}$ & $\mathbf{4 6 2 , 0 8 9}$ & $\mathbf{5 5 4 , 9 5 3}$ & $\mathbf{6 0 5 , 9 5 7}$ \\
\hline Básica & 50,358 & 184,834 & 283,215 & 280,453 & 273,276 & 317,819 & 346,046 \\
\hline Premedia & 104,203 & 124,943 & 125,816 & 121,497 & 114,035 & 141,286 & 152,339 \\
\hline Media & 59,546 & 68,256 & 72,757 & 76,624 & 74,778 & 95,848 & 107,572 \\
\hline
\end{tabular}

Fuente: Información de la Dirección de Planificación. Departamento de Estadística IFARHU.

La inversión social del Programa Beca Universal, representa un monto anual significativo en el presupuesto educativo del país, que supera las asignaciones en otras áreas importantes (Herrera, Torres-Lista, \& Montenegro, 2018) .

En el Cuadro $\mathrm{N}^{\circ} 2$, se puede ver la inversión anual del programa, con un incremento considerable cada año, un presupuesto que en sus inicios representó un presupuesto de 17,128,560 millones y para el 2016 alcanzó la suma de 189,296,975 millones, el mismo sigue aumentando, según refleja el cuadro No.2.

\section{Cuadro No. 2. Beca Universal Concedidas Por El IFARHU, Por Monto} Según Nivel De Estudio: Años 2010 - 2016

\begin{tabular}{|c|c|c|r|r|r|r|r|}
\hline $\begin{array}{c}\text { Nivel de } \\
\text { Estudio }\end{array}$ & $\mathbf{2 0 1 0}$ & $\mathbf{2 0 1 1}$ & $\mathbf{2 0 1 2}$ & $\mathbf{2 0 1 3}$ & $\mathbf{2 0 1 4}$ & $\mathbf{2 0 1 5}$ & $\mathbf{2 0 1 6}$ \\
\hline Total & $\mathbf{1 7 , 1 2 8 , 5 6 0}$ & $\mathbf{4 5 , 0 8 7 , 4 2 0}$ & $\mathbf{5 7 , 4 9 6 , 2 0 0}$ & $\mathbf{8 3 , 4 8 6 , 5 8 0}$ & $\mathbf{7 7 , 1 5 8 , 8 0 0}$ & $\mathbf{1 7 8 , 9 9 8 , 2 7 5}$ & $\mathbf{1 8 9 , 2 9 6 , 9 7 5}$ \\
\hline Básica & $4,028,640$ & $22,057,440$ & $8,709,120$ & $48,735,660$ & $40,999,140$ & $85,518,355$ & $93,256,470$ \\
\hline Premedia & $8,336,240$ & $14,878,200$ & $15,035,580$ & $21,235,260$ & $20,472,900$ & $50,604,995$ & $52,077,620$ \\
\hline Media & $4,763,680$ & $8,151,780$ & $33,751,500$ & $13,515,660$ & $15,686,760$ & $42,874,925$ & $43,962,885$ \\
\hline
\end{tabular}

Fuente: Información de la Dirección de Planificación. Departamento de Estadística IFARHU. 
Invest. pens. crit. (ISSN 1812-3864)

Vol. 7, No. 1, enero- abril 2019

pp. $05-26$

La beca universal como parte de los subsidios sociales, es un tema que amerita un serio debate, no deben ser acciones aisladas, sino articuladas a otros esfuerzos y un acompañamiento del Estado para garantizar el uso adecuado de los recursos. Esto implica, tener estadísticas actualizadas, datos desagregados, metodologías para el seguimiento y evaluación e investigaciones para tener evidencia científica de los problemas de aprendizaje, para intervenir en otros factores.

En ese sentido, los programas y acciones educativas, deben ser el resultado de políticas transparentes, concertadas y con validez científica, se trata de inversiones sociales de recursos que deben mejorar la calidad de vida de la población.

En cuanto a los subsidios Sokol (2016) plantea que, si bien difícilmente atacan la raíz del problema, estas transferencias de recursos tendrán un impacto positivo siempre y cuando se acompañen de medidas que incrementen la productividad en hogares pobres. Esto lleva a la reflexión del programa de subsidio de beca universal. Si bien puede que no resuelva la raíz del problema, no podemos negar el impacto positivo que realiza en diversos hogares panameños, especialmente en las zonas de alto nivel de marginación y vulnerabilidad social. Tal como demuestran Noriega (2017) y Gálvez (2017) la retención de estudiantes en los centros educativos está vinculado a distintos factores psicosociales que intervienen; por tanto, pretender que el programa de beca universal resolverá por si solo el problema es un error; no obstante, en la medida que este acompañado de otros programas su efectividad será mayor.

A propósito de estos factores, vale destacar el estudio regional de la Agencia Interamericana para la Cooperación y el Desarrollo/OEA, donde se plantea que las razones que las escuelas generan para que todos completen su trayectoria educativa en tiempo y forma son múltiples y obedecen a diversos factores que se refuerzan mutuamente. (OEA, 2003). Nos estamos refiriendo a la autoestima académica, motivación escolar, clima del aula, participación y formación ciudadana, hábitos de vida saludable y resiliencia

La beca universal se vincula como factor positivo en la retención de los estudiantes en el sistema; aunque también es importante analizar los otros problemas que este programa busca impactar como la deserción escolar y la disminución del nivel de los fracasos, que nos hablan de la complejidad del problema educativo, pero lo que no podemos como sociedad es dejar de lado los fines de la excelencia y calidad de la educación.

La contradicción inherente del programa, es que por un lado es fundamental garantizar que todos los niños y niñas estén en las escuelas; sin embargo, lo que motiva el estudiar no puede ser el recibir un subsidio económico. Estamos ante lo que Merton (1964) plantea como funciones manifiestas y latentes. El programa busca impactar positivamente en el sistema educativo, para que todos los niños y niñas se mantengan en las escuelas, pero se genera un efecto no deseado; relacionado a lo que motiva estudiar y prepararse.

En los primeros niveles del ciclo educativo, la socialización de los valores y principios son importantes para la vida adulta, los criterios mínimos de recibir beneficios sin exigir un esfuerzo de rendimiento académico, pueden ser un mensaje contradictorio para la niñez y juventud. Precisamente es la contradicción inherente del programa; lograr que todos los niños y niñas no dejen de estudiar, pero la razón que motiva este propósito no puede estar asociada al recibir un beneficio económico. 
Invest. pens. crit. (ISSN 1812-3864)

Vol. 7, No. 1, enero- abril 2019

pp. $05-26$

Cuando el sistema no logra la formación integral de los estudiantes en sus primeros ciclos de estudio, y cumplan mínimamente los aprendizajes; los jóvenes arrastran sus carencias académicas y desarrollo de competencias, limitando su acceso a la educación superior; no logran aprobar las pruebas de conocimientos básicos en las universidades, lo que plantea la necesidad de revaluar el promedio establecido para otorgar el beneficio de la beca.

Urge adoptar políticas educativas que se ajusten a los fines de la educación panameña, de manera integral e inclusiva, con participación de la comunidad educativa y los actores sociales vinculados a la educación. En este sentido, las inversiones sociales, como los subsidios escolares, puede ser una decisión política; pero no puede estar divorciada de los objetivos de la educación panameña y su calidad, de allí la importancia de una evaluación permanente de los resultados de los programas.

La obligación del Estado de garantizar una educación de calidad, no se delega, como tampoco el que deba atender las desigualdades sociales que sufren un significativo grupo de la población estudiantil. "La institucionalidad educativa se ha visto, así, sometida a una serie de transformaciones concebidas y agenciadas, las más de las veces desde fuera de ella, que ha conducido a replantear los alcances de su autonomía, a repensar sus modelos organizativos, a redefinir sus funciones sociales y a incrementar los dispositivos de control y regulación que sobre la escuela ejercen los gobiernos, la política hegemónica, el sistema productivo y los más variados intereses nacionales y transnacionales" (Orozco Cruz, Alfredo, \& Vivian, 2009)

Desde la academia, los centros de investigación, la sociedad civil, las empresas y las instituciones educativas, están convencidos de la urgencia de una transformación de la educación, para una educación de calidad.

Se espera que a partir de este trabajo se logren implementar herramientas metodológicas para poder evaluar el programa y adoptar estrategias para retener a los estudiantes en el sistema, para que completen sus estudios en el tiempo previsto.

\section{Materiales y Métodos}

Metodológicamente, estamos ante un enfoque mixto en donde se desarrollan dos etapas: la primera es de perspectiva cualitativa relacionado al análisis jurídico del programa de la beca universal; el segundo momento, es de corte cuantitativo para medir el grado de impacto que tiene el programa de a beca universal en la retención escolar, pero condicionada a que cumplan el ciclo escolar en el tiempo esperado.

La temporalidad del estudio va del año 1996 al 2017, para poder determinar en su justa dimensión el impacto del programa en relación con años anteriores. 
Invest. pens. crit. (ISSN 1812-3864)

Vol. 7, No. 1, enero- abril 2019

pp. $05-26$

Los datos trabajados, son a nivel censal de la población estudiantil a nivel nacional ${ }^{1}$, específicamente de escuelas oficiales. Toda la información es provista por el Departamento de Estadística del Ministerio de Educación de Panamá y de igual manera fue validada por el mismo.

Para la fase cualitativa, se analiza toda la norma que sustenta el programa, desde el enfoque de la sociología jurídica y ciencias políticas, con perspectiva de derechos humanos. Se triangula la información secundaria bajo el enfoque de Denzin (1970); contrastando y comparando distintas normativas relacionadas, posiciones, análisis crítico, sus vacíos, fortalezas, debilidades y distintas modificaciones que ha sufrido.

En el segundo momento cuantitativo, se propone una variable a medir. Esta variable la hemos denominado, "REDU = Efectividad Educativa" tanto para básica, así como para premedia y media. Debemos entender la variable REDU u efectividad educativa; como la capacidad que tiene el sistema de retener a los estudiantes, pero además de que logren finalizar su ciclo en el tiempo deseado; por ejemplo, básica en 6 años.

Para básica:

En dónde;

$$
R E D U_{b}=\frac{T E B^{a b+5}}{T I B^{a b}} * 100
$$

$a b=$ Año base decidido sobre el cual se desea hacer la evaluación del proceso.

TIB = Total de estudiantes del "ab" que ingresaron al sistema educativo en el nivel básico.

TEB $=$ Total de estudiantes del "ab+5” que egresaron al sistema educativo en el nivel básico.

Para ciclo $7^{\circ}$ a $12^{\circ}$

$$
R E D U_{s}=\frac{T E S^{a b+5}}{T I S^{a b}} * 100
$$

En dónde;

$a b=$ Año base decidido sobre el cual se desea hacer la evaluación del proceso.

TIS = Total de estudiantes del "ab" que ingresaron al sistema educativo en el nivel premedia.

TES = Total de estudiantes del “ab+5” que egresaron al sistema educativo en el nivel media.

Los resultados se expresarán en porcentaje, siendo el 100\% el escenario ideal y el parámetro para indicar si estamos mejorando.

Adicional se propone otra variable complementaria para el análisis. Esta variable la hemos denominado "REZ = Retención escolar de estudiantes pero que no logran terminar el ciclo educativo en el tiempo deseado, también conocidos como estudiantes rezagados". Es decir, estamos ante un grupo de estudiantes que están dentro del sistema educativo, pero su proceso escolar no culminará en el tiempo deseado.

\footnotetext{
${ }^{1}$ Actualmente desde la Universidad Católica Santa María La Antigua, estamos analizando de forma desagregada el comportamiento de los datos en las zonas rurales, urbanas, indígenas. Lo cual esperamos prontamente hacer público.
} 
Para básica:

$$
R E Z_{b}=T I B-T E S_{a b+5}
$$

En dónde;

$a b=$ Año base decidido sobre el cual se desea hacer la evaluación del proceso.

TIB = Total de estudiantes del "ab" que ingresaron al sistema educativo en el nivel básico. TEB $=$ Total de estudiantes del "ab+5" que egresaron al sistema educativo en el nivel básico.

Para ciclo $7^{\circ}$ a $12^{\circ}$

$$
R E Z_{s}=T I S-T E S_{a b+5}
$$

En dónde;

$a b=$ Año base decidido sobre el cual se desea hacer la evaluación del proceso.

TIS = Total de estudiantes del "ab" que ingresaron al sistema educativo en el nivel premedia.

TES $=$ Total de estudiantes del " $a b+5$ ” que egresaron al sistema educativo en el nivel media.

Los resultados se expresarán en cifras absolutas, siendo la reducción de este comparado al ciclo anterior, el escenario ideal y el parámetro para indicar si estamos mejorando.

$\mathrm{Al}$ articular las variables tendremos los siguientes escenarios:

Esquema $\mathrm{N}^{\circ} 1$ Matriz analítica para monitorear el programa de beca universal.

\begin{tabular}{|c|l|c|c|}
\cline { 3 - 4 } \multicolumn{2}{c|}{} & \multicolumn{2}{c|}{ Comportamiento de la Variable "REDU" } \\
\cline { 2 - 4 } \multicolumn{2}{c|}{$\begin{array}{c}\text { Comportamiento de la } \\
\text { Variable "REZ" }\end{array}$} & Incremento $\boldsymbol{\Delta}$ & Disminución $\boldsymbol{\nabla}$ \\
\cline { 2 - 4 } & Disminución $\downarrow$ & $\mathrm{C} \uparrow \boldsymbol{\Delta}$ & $\mathrm{D} \uparrow \boldsymbol{\nabla}$ \\
\hline
\end{tabular}

Fuente: Elaboración propia

Interpretación de la matriz:

Escenario A = Lo hemos denominado, el escenario ideal, en donde hay un claro incremento de la efectividad educativa y al mismo tiempo reducción de estudiantes que no finalizarán en el ciclo escolar deseado.

Escenario $\mathrm{B}$ y $\mathrm{C}=$ En este caso estamos ante un resultado medianamente positivo.

Para el caso del B, estamos ante disminución en cifras absolutas de estudiantes que quedan rezagados; pero disminución de efectividad educativa del sistema en términos $\%$.

Por el otro lado en el C, se incrementa la efectividad educativa del sistema en términos \%; sin embargo, aumento en cifras absolutas de estudiantes que quedan rezagados.

Escenario D = Es el más preocupante y el menos positivo; en donde aumenta en cifras absolutas de estudiantes que quedan rezagados y al mismo tiempo disminución de efectividad educativa del sistema en términos $\%$. 
Invest. pens. crit. (ISSN 1812-3864)

Vol. 7, No. 1, enero- abril 2019

pp. $05-26$

\section{Resultados}

\subsection{Análisis Jurídico De Las Normas Que Regulan El Programa De Beca Universal.}

El Estado panameño, organiza y dirige el servicio público de la educación, y tiene la responsabilidad del cumplimiento de este derecho con principios de solidaridad humana y justicia social, garantizando a los padres el derecho a participar en el proceso educativo de sus hijos, conforme lo consagra la Constitución Politica de la República de Panamá de 1972, y todos sus actos reformatorios, contenidos en el Texto Único de 2004. La Constitución establece la gratuidad de la educación; implica que el Estado debe proporcionar los útiles necesarios para completar su Educación Básica General.

Bajo esta norma constitucional y el amparo del derecho a la Educación contenido en la Convencion de los Derechos del Niño, ratificada por Panamá por medio de la Ley 15 de 6 de noviembre de 1990, publicada en la Gaceta Oficial No. 21667 de 16 de marzo de 1990, y de conformidad a los principios del sistema educativo nacional, se sustenta jurídicamente el Programa de la Beca Universal.

En la Ley 47 de 1946, Orgánica de Educación, se concibe la educación como una inversión social y debe beneficiar a todos los estratos de la sociedad.

El Programa de Beca Universal, está contenido en la Ley No 40 de 23 de agosto de 2010, "Que regula el Programa de Beca Universal y modifica un artículo de la Ley 8 de 2010, relativo al financiamiento del Programa”. Esta Ley tiene vacíos, adolece de objetivos, conceptos, y metodología de evaluación del programa. Durante el proceso de implementación se van delineando sus propósitos y aclarando conceptos.

El Decreto Ejecutivo No.1 de 12 de enero de 2011, reglamenta la Ley 40 de 2010, y logra en gran medida llenar los vacíos de la Ley al definir que La Beca Universal un programa que consiste en un apoyo económico y cuya finalidad es contrarrestar la deserción escolar, elevar considerablemente los índices de inscripción y asistencia de niñas, niños y adolescentes, en los procesos educativos, y señala expresamente cuál es el uso que debe darse al beneficio económico, el cual está destinado para la adquisición de uniformes, libros, útiles, escolares y alimentos para los estudiantes beneficiados.

Es interesante lo señalado en este Decreto, en cuanto a la verificación de rendimiento y asistencia por parte del IFARHU y contempla que la insistencia del beneficiario al centro educativo dará lugar a someter tal hecho a la SENNIAF Y a la Jurisdicción de Niñez y Adolescencia para lo que corresponda en derecho.

A través del Decreto Ejecutivo No. 167 de 13 de mayo de 2012, se modifica el artículo 9 del Decreto Ejecutivo 1 de 2011, para incluir la retención de la Beca Universal, indicando que los pagos retenidos por registro de deficiencia académica son guardados por el IFARHU y entregados en el pago siguiente de la beca, si el estudiante recupera la asignatura. 
Invest. pens. crit. (ISSN 1812-3864)

Vol. 7, No. 1, enero- abril 2019

pp. $05-26$

En la Resolución N0. 320-2013-184 de 25 de marzo de 2013, el Instituto para la Formación y Aprovechamiento de Recursos Humanos (IFARHU), aprobó el Manual de Beca Universal, para incluir los cambios introducidos en los decretos y las leyes que modifican el programa.

La Ley No.14 de 12 de agosto de 2014, adiciona artículos a la Ley 40 aumentando la asignación de la Beca Universal. Después de 4 años de vigencia del programa, es que se incluyen en esta normativa los objetivos de la beca universal como son: 1. Prevenir y contrarrestar la deserción escolar de estudiantes que presenten problemas socioeconómicos, según evaluación del gabinete psicopedagógico, con el otorgamiento de un apoyo económico en sus necesidades escolares básicas; 2. Elevar los índices de inscripción y asistencia escolar dentro de los procesos educativos ; 3.Brindar a oportunidad a los estudiantes de básica, premedia y media, de recibir el beneficio siempre que cuente con el promedio establecido.

Un avance de la Ley 14, fue incorporar la posibilidad de crear los mecanismos de evaluación del programa, señalando que El MEDUCA recibiría los fondos necesarios para tomar las medidas institucionales pertinentes para dar seguimiento al Programa y establece que se deben medir los resultados del programa dos veces al año, al momento del primer pago de la beca y al final de año.

Se define la Beca universal, como apoyo económico que se brinda a estudiantes de básica, premedia y media hasta que culminen sus estudios para lograr los objetivos previstos en esta ley, es decir prevenir y contrarrestar la deserción, elevar la retención y brindar oportunidades, no habla taxativamente de retención, rezago escolar o de prevenir los fracasos, aunque deben impactarse con base al tercer objetivo del programa, dirigido a favorecer a quienes se encuentren en condiciones de vulnerabilidad.

El Decreto Ejecutivo No.55 de 24 de febrero de 2015, y publicada en la Gaceta Oficial No. 27728 del 26 de febrero de 2015, reglamenta la Ley 14 y recoge en sus considerandos los objetivos antes mencionados y de igual forma, reitera que el programa consiste en un apoyo económico.

Las resoluciones aprobadas por el IFARHU, son importantes en la implementación del Programa de Beca Universal y permiten conocer sus objetivos, fines y definiciones, pero el retraso de la reglamentación de la Ley fue un serio inconveniente para su determinar sus resultados.

La Beca Universal, se le define como apoyo económico, sin hacer referencia a ninguno de sus objetivos; aunque en el Manual del Programa, se incorpora una segunda definición más amplia; como una asistencia económica educativa condicionada, que beneficia a todos los estudiantes de sistema público de educación, premedia y Media, así como de los centros educativos particulares, cuya anualidad y mensualidad no supere los mil balboas con 00/100(1,000.00) y su finalidad es prevenir y contrarrestar la deserción escolar. (IFARHU., 2016)

También se explica las tres (3) etapas del sistema educativo donde los estudiantes de la Educación Básica, que tiene una etapa inicial de la Educación Básica General de una duración de 6 años, que comprende de primero hasta sexto grado. La Educación Premedia, que es la etapa final de la Educación Básica General y tiene una duración de 3 años, comprende desde el séptimo grado hasta el noveno y la Educación Media, (Segundo Nivel de Enseñanza), que normalmente tiene una duración de 3 años, y comprende desde el décimo grado hasta el duodécimo. 
Invest. pens. crit. (ISSN 1812-3864)

Vol. 7, No. 1, enero- abril 2019

pp. $05-26$

Se considere que el programa responde a una serie de actividades que corresponden a un objetivo gubernamental y no menciona que forme parte de la política educativa.

Con la modificación introducida por la Ley 14 de 2014, se aumentan los montos de la beca pagada anualmente de la siguiente forma: $\$ 270$ para cada escolar de nivel básico; $\$ 360$ para los de educación premedia y $\$ 450$ para los estudiantes de educación media, cuyos beneficios se extienden en las escuelas y colegios particulares, mediante la Ley 389 de marzo de 2017.

Aunque la Ley 40 no incorpora principios, fines y funciones; las normas procedimentales, antes comentadas permiten establecer parámetros para entender su motivación para la adopción de este subsidio educativo.

En la Ley 389 de 22 de marzo de 2017, extiende el beneficio de la beca universal a los alumnos de escuelas privadas con gastos de matrícula y anualidades que no excedan de 2,000 dólares anuales.

A pesar de las distintas modificaciones para llenar los vacíos iniciales de Ley 40 que regula el Programa, las mismas se convierten en limitante en la elaboración de los diagnósticos e instrumentos para evaluar el impacto del programa.

Cuadro No.3. Ley 40 de 2010, que regula el programa de la Beca Universal.

En el Art. 1 Se establece que el IFARHU con la colaboración del MEDUCA ejecutara de manera progresiva el programa. La entrada en vigencia del programa inicia en el segundo semestre del 2010, en las Provincias de Bocas del Toro y la Comarca Ngäbe Buglé. En este artículo encontramos tres aspectos: Entidad competente del programa, entrada en vigencia y el área geográfica para iniciar el programa.

El Art. 2. Contempla la Asignación de 180.00 balboas, pagados según el calendario establecido por Decreto Ejecutivo. El acudiente está obligado a acudir personalmente al centro oficial o particular a retirar el boletín y el monto de la beca. Se establece el monto de la Beca Universal, el calendario de pago y obligación del acudiente de retirar boletín y la beca. En el Ar.3. Se encuentran los requisitos para que el IFARHU entregue la Beca Universal a los estudiantes.

$\checkmark$ Sea alumno del subsistema regular de educación.

$\checkmark \quad$ No cuente con beneficio educativo estatal.

$\checkmark$ Haya aprobado el año escolar anterior al otorgamiento de la beca y cuente con un promedio general mínimo de 3.0 o su equivalente, cuando se trate de estudiante de educación básica.

$\checkmark$ No hay reprobado asignaturas en el año escolar anterior al otorgamiento de la Beca, ni tenga asignaturas pendientes de años anteriores y cuente con un promedio académico mínimo de 3.0 o su equivalente en cada asignatura, cuando se trate de estudiantes de media y premedia.

El Art.4. Señala que el otorgamiento de la Beca Universal es hasta la culminación de los estudios de educación media y no hay restricción en cuanto al número de beneficiarios en un mismo núcleo familiar. Corresponde al MEDUCA, entregar al IFARHU la lista oficial de estudiantes matriculados en los centros educativos oficiales y particulares.

En el Art.5. Establece que si al momento de recibir el pago, conforme al calendario anual, los estudiantes de educación premedia y media registre deficiencia académica, por lo menos en una asignatura del año lectivo, el pago será retenido y sólo será entregado si en la siguiente fecha de pago, evidencia haber recuperado la asignatura o asignaturas en que registró deficiencia.

Los estudiantes de educación básica, para mantener el beneficio de la beca universal, deben tener un promedio mínimo de 3.0 o equivalente por bimestre. Si el promedio es inferior, el pago es retenido y se entregará en la siguiente fecha de pago, siempre haya alcanzado el promedio general. Es decir que el índice académico mínimo que se requiere, es la calificación de 3.0 que ya el sistema contempla para ser aprobado, Este es uno de los principales argumentos de rechazo de la beca.

El Art.6. señala que el IFARHU puede cancelar la beca universal, en los siguientes casos:

$\checkmark \quad$ No hacer uso de la beca en el año lectivo correspondiente 
Invest. pens. crit. (ISSN 1812-3864)

Vol. 7, No. 1, enero- abril 2019

pp. 05-26

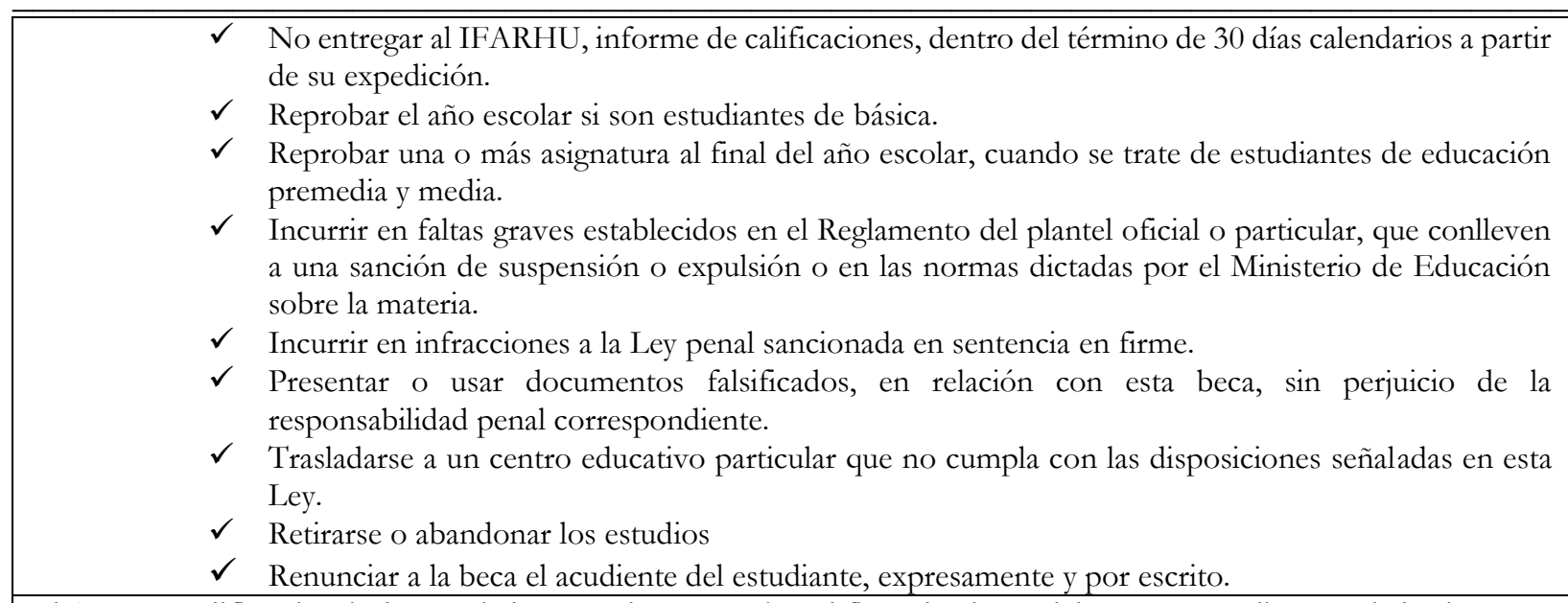

El Art. 7. Modifica el artículo 169 de la Ley 8 de 2010, sobre el financiamiento del Programa y dice que de los ingresos adicionales que se recauden con el Impuesto sobre la Transferencia de Bienes Corporales Muebles y la prestación de servicios (ITBMS)previsto en la ley, financiara entre otros, el costo total del Programa de Beca Universal, para todos los estudiantes de los centros educativos, oficiales y particulares del país, debidamente reconocidos por el Ministerio de Educación, desde la educación básica hasta la educación media, con base al rendimiento académico.

Para que los estudiantes de escuelas particulares se beneficien de la beca universal, la suma de la matrícula y las mensualidades no deberá exceder los mil balboas, (1,000.00) anuales. En el 2017 aumenta el tope a dos mil balboas anuales.

Se reitera lo establecido en el artículo 1 que el programa será ejecutado por el IFARHU con la colaboración del Ministerio de Educación.

En el Art. 8. Señala que el Órgano Ejecutivo a través del Ministerio de Educación Reglamentará la Ley. El Art.9. Esta ley modifica el artículo 169 de la Ley 8 de 15 de marzo de 2010.

En el Art. 10. En relación a la entrada en vigencia de la Ley, se establece al día siguiente de su promulgación.

Fuente: Elaboración propia a partir del análisis de la Ley 40 de 2010.

La fuente de financiamiento de la Beca Universal está contemplada en la Ley 8 de 15 de marzo de 2010, "Que reforma el Código Fiscal, adopta medidas fiscales y crea el Tribunal Administrativo Tributario", fue publicada en la Gaceta Oficial No 26489-A, de la misma fecha.

En la Ley 40 de 2010, se introduce un cambio al artículo 169 de la Ley 8 sobre el financiamiento de la Beca Universal; cuyos fondos debían cubrir el costo total del programa para todos los estudiantes de educación básica, hasta la educación media, (antes primer grado hasta el noveno grado) donde se dice que la suma de la matrícula no debe exceder de los mil balboas. Antes de esta modificación el monto mensual a pagar a los acudientes de las escuelas particulares no debía superar los 200.00 balboas.

Las distintas disposiciones jurídicas de la Beca Universal, particularmente los decretos que la reglamentan arrojan luces sobre los objetivos y fines del programa, que se amplían, con la finalidad de poder analizar el impacto de la implementación de la Beca Universal, a saber:
$\checkmark$ Contrarrestar la deserción escolar.
$\checkmark$ Elevar considerablemente los índices de matriculación
$\checkmark$ Asistencia en los procesos educativos
$\checkmark$ Mejorar la calidad educativa
$\checkmark$ Prevenir la entrada en el mercado de trabajo ilegal
$\checkmark$ Participación en actividades delictivas 
Invest. pens. crit. (ISSN 1812-3864)

Vol. 7, No. 1, enero- abril 2019

pp. $05-26$

La Ley 389 de 22 de marzo de 2017, modifica la Ley 40 de 2010, ampliando el beneficio a los estudiantes de escuelas particulares, con lo cual, dependiendo el nivel de enseñanza, se establece el monto de B/270 balboas para estudiantes de la educación básica, B/360.00 balboas para estudiantes de la educación premedia y B/450.00 para estudiantes de la educación media.

No se ha encontrado documentos que sustenten un diagnóstico de la situación social de los estudiantes o estudio a profundidad para adoptar el programa, como tampoco mecanismos de monitoreo y evaluación, de allí la importancia de este estudio, como parte de las estrategias para una educación de calidad. El análisis apunta a repensar los criterios aplicables a las escuelas públicas y las particulares, porque no es que se niegue el apoyo económico, pero al final lo que debe prevalecer es el objetivo de garantizar la calidad de la educación y el acceso a este derecho con igualdad de oportunidades y equidad.

Los subsidios estatales reciben cuestionamientos por las altas sumas que representan, cuando no se informa de sus resultados; en medios de comunicación han aparecido publicaciones, donde especialistas en materia educativa se manifiestan sobre estas inversiones; el Rector de la USMA Dr. Planells, expresa que en estos programas "se deben establecer prioridades porque el país no tiene recursos ilimitados” (Arcia Jaramillo, 2017)

Diógenes Sánchez, de la Asociación de Profesores, considera que la Beca Universal, el promedio debería ser más alto, porque no se está promoviendo la meritocracia. (Sánchez, 2016)

Para el 2017 estos dineros ya son retirados en su mayoría por los estudiantes a través del Sistema de Tarjeta Clave Social, al que están afiliadas 392 centros educativos en todo el país, no existen información cómo el sistema educativo orienta en el uso de los recursos para los fines educativos.

El programa de beca universal es positivo, cuenta con un marco jurídico, pero su conceptualización criterios y requisitos, debe incluir otras estrategias educativas y mecanismos de evaluación científicamente validados, que permitan cumplir sus objetivos.

\subsection{Efectividad de retención condicionada al cumplimiento del ciclo escolar en el tiempo previsto.}

Conocer los efectos de la beca universal, en la retención de estudiantes que cumplen todo su ciclo educativo, busca identificar la efectividad del sistema, que permita introducir cambios, adoptar un sistema de seguimiento y evaluación a la trayectoria escolar de los estudiantes, introducir innovaciones pedagógicas, fortalecer el programa, así como la creación de índice de impacto, y se propone modificar su nombre, como Programa de Apoyo Económico de Oportunidad Educativa (PAE), con otros parámetros de rendimiento académico, objetivos y responsabilidad familiar.

Como hemos señalado, la beca universal, creada por la Ley 40 de 2010, es positiva pese a que surge con vacíos y se hacen distintas modificaciones, adiciones y reglamentaciones, sin lograr mecanismos científicos de seguimiento sobre sus resultados, como tenía previsto a través de la creación de los equipos multidisciplinarios y departamentos de orientación en cada escuela. Objetivamente, no se puede atribuir a este programa la solución de todos los complejos factores y problemas (individuales, 
Invest. pens. crit. (ISSN 1812-3864)

Vol. 7, No. 1, enero- abril 2019

pp. $05-26$

familiares, sociales y económicos) presentes en la falta de retención, en la deserción o en fracasos escolares.

El problema educativo requiere de respuestas integrales y articuladas para lograr la permanencia con éxito de los estudiantes en todos los niveles; acciones como horario extendido, tutorías; orientación psicopedagógica, alternativas tecnológicas motivadoras y acompañamiento entre pares, son algunas de las que se pueden implementar para tener los resultados esperados.

La escuela debe ser capaz de lograr que el estudiante logre más que un conocimiento académico, internalice el aprender a aprender, el aprender a hacer, el aprender a ser y el aprender a vivir en comunidad, principios que la UNESCO, ha promulgado en una educación para todos. (EPT).

El problema central de los sistemas educativos latinoamericanos es la escasa capacidad de retención de niños y particularmente de jóvenes. La información disponible indica que actualmente cerca del $37 \%$ de los adolescentes latinoamericanos entre 15 y 19 años de edad abandonan la escuela a lo largo del ciclo escolar, y casi la mitad de ellos lo hacen tempranamente, antes de completar los años de obligatoriedad. (Espínola, 2002)

De acuerdo al Cuadro $\mathrm{N}^{\circ}$ 4, los resultados demuestran que históricamente (1996-2012), el país ha mantenido una positiva cifra de retención de los estudiantes de básica, entre los 74\% al 87\%; pero en el 2012, con la implementación de la Beca Universal y otros programas se logra retener al 94\% de los estudiantes. Sin embargo, en la educación Premedia y Media, (antes secundaria), la retención de estudiantes no alcanza el 50\%, con excepción del año 2000. Pero luego de terminar la implementación del programa se demuestra un impacto significativo en el grupo de niños y niñas del 2012 al 2017, logrando una retención con eficiencia escolar del $51 \%$.

\section{Cuadro $\mathrm{N}^{\circ} .4$ comparación del \% de efectividad educativa en básica, premedia-media de escuelas oficiales de Panamá. 1996-2017}

\begin{tabular}{|c|c|c|}
\hline Ciclo & $\begin{array}{c}\% \text { de efectividad educativa en } \\
\text { básica }\left(1^{\circ}-6^{\circ}\right)\end{array}$ & $\begin{array}{l}\% \text { de efectividad educativa en } \\
\text { el ciclo }\left(7^{\circ}-12^{\circ}\right)\end{array}$ \\
\hline $96-01$ & $74 \%$ & $47 \%$ \\
\hline $97-02$ & $75 \%$ & $48 \%$ \\
\hline $98-03$ & $77 \%$ & $47 \%$ \\
\hline $99-04$ & $77 \%$ & $49 \%$ \\
\hline 2000-05 & $77 \%$ & $50 \%$ \\
\hline 2001-06 & $76 \%$ & $49 \%$ \\
\hline 2002-07 & $76 \%$ & $46 \%$ \\
\hline 2003-08 & $78 \%$ & $45 \%$ \\
\hline 2004-09 & $78 \%$ & $44 \%$ \\
\hline 2005-10 & $81 \%$ & $43 \%$ \\
\hline 2006-11 & $81 \%$ & $43 \%$ \\
\hline $2007-12$ & $82 \%$ & $42 \%$ \\
\hline 2008-13 & $85 \%$ & $43 \%$ \\
\hline 2009-14 & $86 \%$ & $45 \%$ \\
\hline 2010-15 & $87 \%$ & $45 \%$ \\
\hline 2011-16 & $87 \%$ & $23 \%$ \\
\hline $2012-17$ & $94 \%$ & $51 \%$ \\
\hline
\end{tabular}

Fuente: Elaboración Propia a partir de los datos proporcionados por el Departamento de Estadística del MEDUCA.2017. 
Invest. pens. crit. (ISSN 1812-3864)

Vol. 7, No. 1, enero- abril 2019

pp. $05-26$

En la gráfica $\mathrm{N}^{\circ} .1$ podemos ver la comparación en todos los niveles de básica, premedia y media, que muestra claramente el comportamiento de la efectividad del sistema educativo en cuanto a la retención y cumplimiento de los estudiantes de su ciclo escolar en el tiempo establecido, en ambos niveles es positivo, aunque como se observa más efectivo en la educación básica con un 94\% en el ciclo escolar 2012-2017, mejorando un 7\% en relación al ciclo anterior del 2011-2016.

Por otro lado, los datos revelan que el inicio del Programa de beca universal, para la premedia y media, se empieza a evidenciar el impacto, a partir del ciclo 2012-2017, mejorando de un 23\% de eficiencia escolar a un 51\%, coincidiendo con las modificaciones introducidas al programa que clarifican sus objetivos.

Hay que destacar que luego de 17 años, se logra sobrepasar el 50\% en retención de estudiantes, condicionada a que terminen sus estudios en el ciclo esperado, evidenciando un impacto positivo en el sistema educativo.

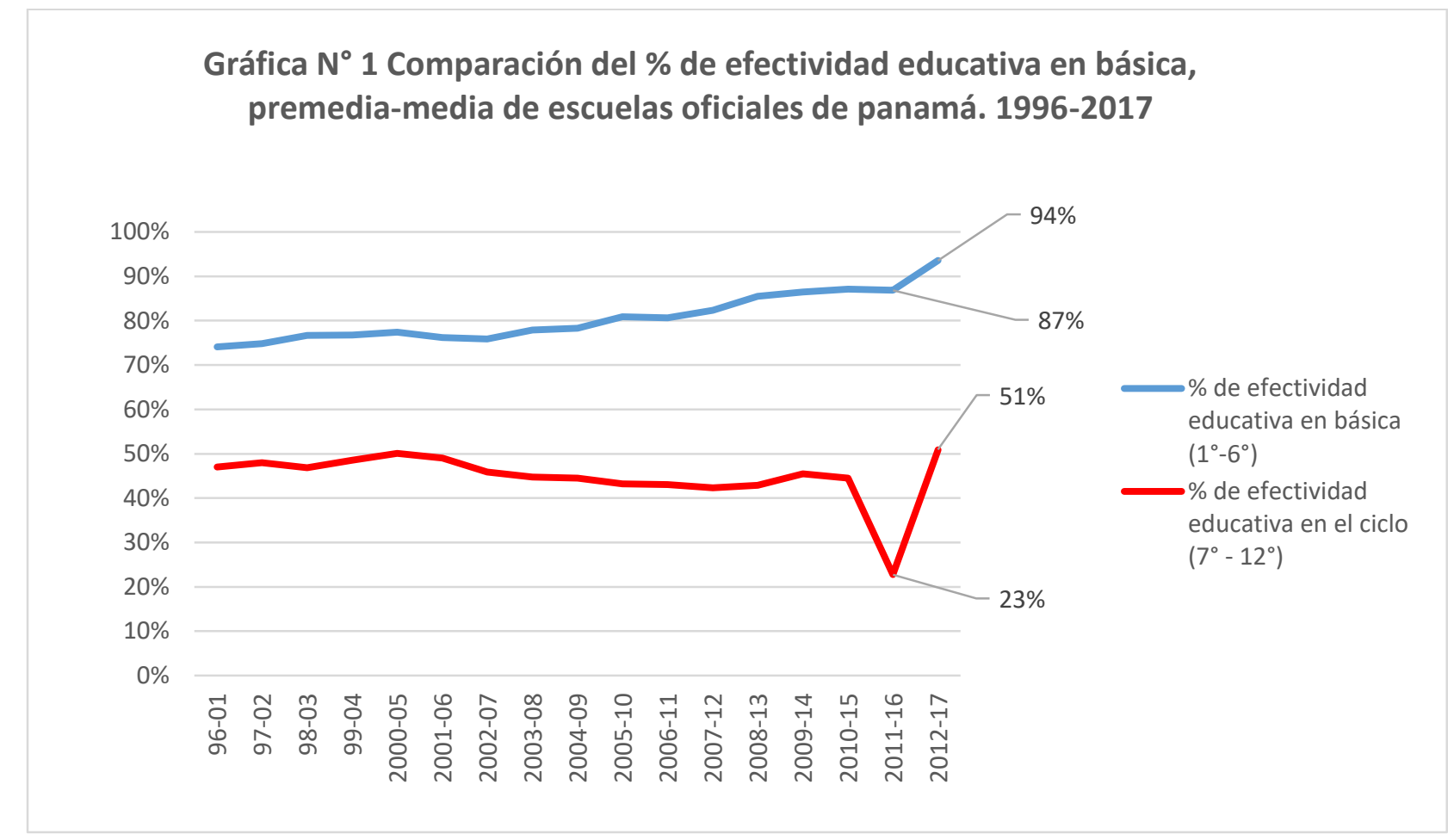

Fuente. Elaboración Propia a partir de los datos proporcionados por el Departamento de Estadística del MEDUCA.2017.

La mayor retención se produce en la educación básica; es importante destacar que en la básica el papel de los padres en esta etapa de la educación de sus hijos e hijas es relevante. En básica los tutores o acudidos se involucran más en la educación, pero existen distintos factores que inciden positivamente en los resultados de la retención en este nivel.

La autoestima y motivación escolar son importantes en las estrategias de retención escolar; pero se destaca que, en la transición de la básica a la secundaria, los estudiantes experimentan un descenso en la motivación del aprendizaje en general. (Noriega Martinez, 2017). 
Invest. pens. crit. (ISSN 1812-3864)

Vol. 7, No. 1, enero- abril 2019

pp. $05-26$

\begin{tabular}{|c|c|c|c|c|c|c|}
\hline \multicolumn{7}{|c|}{$\begin{array}{c}\text { Cuadro } \mathrm{N}^{\circ} .5 . \text { Comparación por género del \% de efectividad educativa en básica, de } \\
\text { escuelas oficiales de Panamá. 1996-2017 }\end{array}$} \\
\hline Ciclo & $\begin{array}{l}\text { Estudiantes } \\
\text { Rezagados } \\
\text { en Cifras } \\
\text { Absolutas } \\
\text { del Ciclo } \\
\text { Básica }\end{array}$ & $\begin{array}{c}\% \text { de } \\
\text { efectividad } \\
\text { educativa en } \\
\text { básica }\left(1^{\circ}-6^{\circ}\right)\end{array}$ & $\begin{array}{l}\text { Estudiantes } \\
\text { Niños } \\
\text { Rezagados en } \\
\quad \text { Cifras } \\
\text { Absolutas del } \\
\text { ciclo Básica }\end{array}$ & $\begin{array}{c}\% \text { de } \\
\text { efectividad } \\
\text { educativa de } \\
\text { niños en } \\
\text { básica }\left(1^{\circ}-6^{\circ}\right)\end{array}$ & $\begin{array}{c}\text { Estudiantes } \\
\text { Niñas } \\
\text { Rezagados en } \\
\text { Cifras Absolutas } \\
\text { del ciclo Básica }\end{array}$ & $\begin{array}{l}\% \text { de efectividad } \\
\text { educativa de niñas } \\
\text { en básica }\left(1^{\circ}-6^{\circ}\right)\end{array}$ \\
\hline $96-01$ & 18080 & $74 \%$ & 10367 & $72 \%$ & 7713 & $76 \%$ \\
\hline $97-02$ & 17532 & $75 \%$ & 10022 & $73 \%$ & 7510 & $77 \%$ \\
\hline $98-03$ & 16038 & $77 \%$ & 9215 & $75 \%$ & 7093 & $78 \%$ \\
\hline 99-04 & 16347 & $77 \%$ & 9305 & $75 \%$ & 7042 & $79 \%$ \\
\hline $2000-05$ & 15878 & $77 \%$ & 8726 & $76 \%$ & 7152 & $79 \%$ \\
\hline 2001-06 & 16712 & $76 \%$ & 9295 & $75 \%$ & 7417 & $78 \%$ \\
\hline $2002-07$ & 17929 & $76 \%$ & 10209 & $74 \%$ & 7720 & $78 \%$ \\
\hline 2003-08 & 16979 & $78 \%$ & 9822 & $76 \%$ & 7157 & $80 \%$ \\
\hline 2004-09 & 16675 & $78 \%$ & 9293 & $77 \%$ & 7382 & $80 \%$ \\
\hline 2005-10 & 13859 & $81 \%$ & 7902 & $79 \%$ & 5957 & $83 \%$ \\
\hline $2006-11$ & 14749 & $81 \%$ & 8235 & $79 \%$ & 6514 & $82 \%$ \\
\hline $2007-12$ & 13303 & $82 \%$ & 7440 & $81 \%$ & 5863 & $84 \%$ \\
\hline 2008-13 & 10385 & $85 \%$ & 5955 & $84 \%$ & 4430 & $87 \%$ \\
\hline $2009-14$ & 9239 & $86 \%$ & 5175 & $86 \%$ & 4064 & $87 \%$ \\
\hline 2010-15 & 8886 & $87 \%$ & 5115 & $86 \%$ & 3771 & $88 \%$ \\
\hline 2011-16 & 8772 & $87 \%$ & 5073 & $85 \%$ & 3699 & $88 \%$ \\
\hline $2012-17$ & 4161 & $94 \%$ & 2803 & $92 \%$ & 1358 & $96 \%$ \\
\hline
\end{tabular}

Fuente: Elaboración Propia a partir de los datos proporcionados por el Departamento de Estadística del MEDUCA.2017.

En el Cuadro $\mathrm{N}^{\circ} 5$ se demuestra que existe una diferencia de género en cuanto a la retención y cumplimiento del ciclo en la educación básica. Mientras se retiene a 92\% de los niños, las niñas logran un $96 \%$, siendo el total de retención en la básica al 2012 de $94 \%$, es decir que a partir del año 2012 se amplía de forma positiva los resultados alcanzados, en comparación del periodo 1996 al 2004 que estaba por debajo del $80 \%$ de retención y cumplimiento del ciclo de básica. Con la entrada del programa en el 2010 se incrementa al 87\%.

Adicional al apoyo económico de la beca universal, existen otros factores que intervienen en la retención del ciclo escolar, por razones de género, como la autoestima de las niñas, motivación, clima escolar y resiliencia.

En cuanto a los rezagos educativos, para los efectos de este análisis, se refiere a los estudiantes que se mantienen en el sistema, pero que no finalizan sus estudios en el tiempo establecido por distintas razones; de índole personal, familiar, socioeconómico, social, pedagógico, y que debe ser parte de las evaluaciones, porque son el semillero de la deserción escolar, por lo que hay que tomar las medidas para mejorar los entornos de aprendizaje, e involucrar a las madres y padres de familia en las actividades de aprendizaje de sus hijos e hijas. 
Invest. pens. crit. (ISSN 1812-3864)

Vol. 7, No. 1, enero- abril 2019

pp. $05-26$

Sí se pretende prevenir y reducir la deserción escolar e impactar los fracasos, se debe dar seguimiento a los estudiantes con rezago educativo. Una intervención oportuna de recuperación de asignaturas que les representan dificultades; la formación continua de los docentes en la transformación de métodos y pedagogías inadecuadas que profundizan el problema; es responsabilidad del sistema educativo y de la comunidad resolverlos. Lograr la permanencia escolar, es importante, pero tiene que garantizar el derecho a una educación de calidad, pertinente, como tema de Estado y compromiso de todos.

Las estadísticas del MEDUCA, sobre los estudiantes rezagados en cifras absolutas en el inicio del programa de la beca universal, muestran un impacto positivo; lo que indica que sin este apoyo económico estos estudiantes podrían ser parte de las cifras de deserción.

En el cuadro No.5 se observa que, en el ciclo del 2011, en cifras absolutas eran 8,772 estudiantes rezagados en básica; mientras que en el ciclo del 2010 eran 8,886. En cuanto al ciclo de 2012 se reduce a 4,161 estudiantes rezagados, representando más de la mitad de las estudiantes que el ciclo anterior y en cuanto al género, los niños rezagados eran superior a las niñas, representando 2,803 niños; mientras las niñas rezagadas fueron 1,358.

Una educación de calidad debe favorecer el desarrollo de niños resilientes, con capacidad de afrontar cualquier situación adversa, con autoestima y autoconfianza; que es otro factor positivo en el aprendizaje y la retención y cumplimiento del ciclo escolar. Según Rivera, en un estudio sobre resiliencia en las escuelas panameñas, los niños, "suelen responder adecuadamente frente a los problemas cotidianos, son más flexibles y sociales, predomina la actitud racional, poseen buena capacidad de autocontrol y autonomía” (Rivera Camacho, 2018).

La participación de padres, madres y acudientes es otro factor esencial en todo el proceso de aprendizajes, de la niñez y la adolescencia. "Los padres juegan un papel importante en la permanencia de los niños en los planteles, los problemas de pareja, la estructura inestable de la familia y la falta de participación de los padres en la formación de los niños son algunos de los factores que determinan el abandono de los niños de la escuela”. (Sarmiento Goméz, 2006). 
Invest. pens. crit. (ISSN 1812-3864)

Vol. 7, No. 1, enero- abril 2019

pp. $05-26$

\section{Cuadro $\mathrm{N}^{\circ}$. 6. Comparación por género del \% de efectividad educativa en el ciclo de educación premedia y media de escuelas oficiales de Panamá. 1996-2017}

\begin{tabular}{|c|c|c|c|c|c|c|}
\hline Ciclo & $\begin{array}{c}\text { Estudiantes } \\
\text { Rezagados en } \\
\text { Cifras } \\
\text { Absolutas del } \\
\text { Ciclo } \\
\left(7^{\circ}-12^{\circ}\right)\end{array}$ & $\begin{array}{c}\% \text { de } \\
\text { efectividad } \\
\text { educativa en } \\
\text { el ciclo } \\
\left(7^{\circ}-12^{\circ}\right)\end{array}$ & $\begin{array}{c}\text { Estudiantes Niños } \\
\text { Rezagados en } \\
\text { Cifras Absolutas } \\
\text { del ciclo }\left(7^{\circ}-12^{\circ}\right)\end{array}$ & $\begin{array}{c}\% \text { de } \\
\text { efectividad } \\
\text { educativa de } \\
\text { niños en el ciclo } \\
\left(7^{\circ}-12^{\circ}\right)\end{array}$ & $\begin{array}{c}\text { Estudiantes } \\
\text { Niñas Rezagados } \\
\text { en Cifras } \\
\text { Absolutas del } \\
\text { ciclo }\left(7^{\circ}-12^{\circ}\right)\end{array}$ & $\begin{array}{c}\% \text { de } \\
\text { efectividad } \\
\text { educativa de } \\
\text { niñas en el } \\
\text { ciclo }\left(7^{\circ}-12^{\circ}\right)\end{array}$ \\
\hline $96-01$ & 23485 & $47 \%$ & 13638 & $42 \%$ & 9853 & $53 \%$ \\
\hline $97-02$ & 22910 & $48 \%$ & 13258 & $43 \%$ & 9652 & $54 \%$ \\
\hline 98-03 & 24672 & $47 \%$ & 13991 & $42 \%$ & 10681 & $52 \%$ \\
\hline $99-04$ & 23862 & $49 \%$ & 13564 & $44 \%$ & 10298 & $54 \%$ \\
\hline 2000-05 & 23631 & $50 \%$ & 13758 & $45 \%$ & 9873 & $56 \%$ \\
\hline 2001-06 & 24734 & $49 \%$ & 14807 & $42 \%$ & 9927 & $57 \%$ \\
\hline $2002-07$ & 27555 & $46 \%$ & 15675 & $41 \%$ & 11880 & $51 \%$ \\
\hline 2003-08 & 29103 & $45 \%$ & 16689 & $39 \%$ & 12414 & $51 \%$ \\
\hline 2004-09 & 28620 & $44 \%$ & 16253 & $39 \%$ & 12367 & $50 \%$ \\
\hline 2005-10 & 29859 & $43 \%$ & 17167 & $38 \%$ & 12692 & $50 \%$ \\
\hline 2006-11 & 30576 & $43 \%$ & 17516 & $37 \%$ & 13060 & $49 \%$ \\
\hline 2007-12 & 32393 & $42 \%$ & 18619 & $37 \%$ & 13770 & $48 \%$ \\
\hline 2008-13 & 33828 & $43 \%$ & 19101 & $38 \%$ & 14727 & $48 \%$ \\
\hline 2009-14 & 32324 & $45 \%$ & 18618 & $40 \%$ & 13706 & $52 \%$ \\
\hline 2010-15 & 35502 & $45 \%$ & 20989 & $38 \%$ & 14513 & $51 \%$ \\
\hline 2011-16 & 48474 & $23 \%$ & 27141 & $19 \%$ & 20973 & $29 \%$ \\
\hline $2012-17$ & 31917 & $51 \%$ & 19305 & $44 \%$ & 12612 & $59 \%$ \\
\hline
\end{tabular}

Fuente. Elaboración propia a partir de los datos del MEDUCA.2017.

Las niñas en la educación premedia y media, alcanzan un 59\% de retención y cumplimiento del ciclo en el tiempo esperado; mientras que los niños un 51\%; pero lo importante es que el sistema educativo tanto para los niños y las niñas beneficiarias del programa de la beca universal, logra a partir del 2012 aumentar los niveles de retención con relación al ciclo anterior, del 23\% al 51\%.

En cuanto a los estudiantes rezagados en cifras absolutas en el ciclo del 2012, es de 31,917; pero con tendencia a la baja en comparación al ciclo anterior de 48,474 estudiantes rezagados. Los datos por género, igual que en la básica muestran que las niñas están aprovechando más los beneficios de la beca universal, son 12,612 niñas en cifras absolutas, mientras que los niños son 19,305.

Las cifras tanto en retención y cumplimiento del ciclo escolar en el tiempo; y el rezago escolar, muestran un impacto positivo del programa de beca universal, a pesar que tenemos que avanzar en políticas públicas, eficientes, eficaces, integrales, multiculturales, con equidad de género, concertadas, pertinentes a las necesidades de este siglo; porque cada estudiante presenta una realidad individual y socioeconomica que interviene en las condiciones iniciales de su proceso de aprendizaje, en este sentido, los programas sociales son respuesta para que los más vulnerables, los que mantienen una 
Invest. pens. crit. (ISSN 1812-3864)

Vol. 7, No. 1, enero- abril 2019

pp. $05-26$

posición desventajosa, en el acceso a sus derechos tengan las mismas oportunidades a una educación de calidad.

En todos los niveles del sistema educativo, el alto los índices de inasistencia, es un aldabonazo de la necesidad de intervenir oportunamente para ofrecer orientación al estudiante y su familia; brindar apoyo psicopedagógico que minimice la posibilidad de deserción, reforzar o crear programas que garanticen la retención escolar; en algunos casos, los estudiantes pueden desertar temporalmente por circunstancias individuales, familiares o socioeconómicas; pero retoman sus estudios, son situaciones que con instrumentos adecuados de evaluación puede el sistema ayudar a prevenir, en otros casos, es más complejo, porque no regresan al sistema, pueden ser los nuevos Ninis, y lo que es más grave, jóvenes que tienen mayor riesgo de incorporarse a grupos al margen de la Ley. Lo que es inaceptable es no evitar que estudiantes dentro del sistema, con problemas muchas veces generados por factores vinculados al ambiente escolar, no reciban la atención para completar su educación.

Los resultados obtenidos son alentadores, muestran una variable de efectividad con perspectiva de género, es decir, que las niñas, tanto en la educación básica, media y premedia están aprovechando más el beneficio del programa de la beca universal, en cuanto a existe una retención de las estudiantes que lograron cumplir en el tiempo su ciclo escolar.

En los resultados de la educación básica, la beca universal es uno de los factores que incide positivamente en la disminución de la deserción escolar, pero no tiene los mismos resultados en la premedia y media. Lo que lleva a la reflexión de la necesidad de identificar que provoca que en básica el programa sea más efectivo que en el ciclo de $7^{\circ}$ a $12^{\circ}$.

\section{Conclusiones y recomendaciones}

La beca universal consiste en un apoyo educativo positivo, aprobado en el 2010 y modificado a través de distintas leyes, decretos y reglamentaciones que lo sustentan; pero que presenta vacíos, contradicciones y cuyos requisitos son cuestionados, especialmente en la calificación mínima de 3.0 y la ausencia de una evaluación científica de su impacto.

Los resultados de este estudio demuestran que históricamente (1996-2012), el país ha mantenido una positiva cifra de retención de los estudiantes de básica, entre los 74. \% al 87 \%; pero en el 2012, con la implementación de la Beca Universal y otros programas. Se logra retener al 94\% de los estudiantes. Es decir, que el programa si tienen un impacto favorable en mantener al estudiantado durante el ciclo de estudio, en el tiempo establecido.

Existe una variable de efectividad del programa con perspectiva de género; las niñas tanto en la educación básica, media y premedia están aprovechando más el beneficio del programa de la beca universal, en cuanto a que existe una mayor retención de las estudiantes que lograron cumplir en el tiempo su ciclo escolar.

Se identifican otros factores que impactan los resultados en la retención del ciclo escolar por razones de género, además del apoyo económico de la beca universal; como son la autoestima de las niñas, la motivación, clima escolar y resiliencia, aspectos que deben ser priorizados en cualquier estrategia para enfrentar el problema de la retención con efectividad. 
Invest. pens. crit. (ISSN 1812-3864)

Vol. 7, No. 1, enero- abril 2019

pp. $05-26$

Los estudiantes con rezago escolar que se mantienen en el sistema, pero que no finalizan sus estudios en el tiempo establecido, requieren de programas de apoyo para superar las dificultades que pueden ser de distinta índole; personal, familiar, socioeconómico, social, pedagógico.

Lo que es importante destacar, que en el ciclo del 2011 a inicio del programa se empieza a dar una ruptura positiva en los datos; estamos hablando que sin la beca podrían ser parte de las cifras de deserción escolar.

El programa genera contradicciones en cuanto no estimula la excelencia educativa y no resiste continuar de forma aislada tratando de enfocarse en cumplir las metas de disminuir los niveles de deserción; debe buscar la retención efectiva en el cumplimiento del ciclo escolar, así como evitar los rezagos. Es buena la retención, pero al mismo tiempo debe tener contrapesos en los valores de la persona; se requiere reforzar los contenidos de aprendizaje en las áreas humanísticas, las ciencias sociales, empoderar y formar una ciudadanía sustantiva.

Se tiene que analizar el financiamiento del Programa de Beca Universal, para todos los estudiantes de los centros educativos, oficiales y particulares, atendiendo los objetivos y que cambios deben introducirse en las escuelas públicas y particulares.

Se recomienda modificar el nombre de "Beca Universal" que es una distorsión que genera rechazo, se propone se denomine Programa de Apoyo Económico de Oportunidad Educativa (PAE), pero estableciendo nuevos criterios académicos, ampliando sus objetivos, incluyendo metodologías de seguimiento y evaluación de resultados, la participación de la comunidad educativa, así como la articulación de este subsidio a otras estrategias existentes que permitiría hacer un mejor uso de la inversión social.

Hay que aprobar una metodología de evaluación para medir el impacto al programa por región escolar atendiendo las condiciones psicosociales de los estudiantes en el nivel de básica, premedia y media, los indicadores de retención efectiva, rezago, deserción y fracaso escolar; y el compromiso de las familias en el proceso educativo.

Todo padre, madre o acudiente de un estudiante beneficiado con la beca universal debe recibir una inducción del uso de los recursos, suscribir un compromiso de rendimiento académico para evitar que sus hijos e hijas fracasen o deserten del sistema escolar.

Existen distintas oportunidades de apoyo económico para estudiar que ofrece el IFARHU entre ellas: becas a puesto distinguido por concurso general, en bellas artes, por convenios culturales y para estudiantes sobresalientes en eventos académicos y culturales; también se incluyen programas de asistencia para la población en situación de vulnerabilidad y riesgo; asistencia a discapacitados, para los corregimientos de mayor pobreza asistencia económica educativa, asistencia para la erradicación trabajo infantil y asistencia colectiva.

Fortalecer una estrecha coordinación con las universidades, en materia de investigaciones sociales que generen los conocimiento científico que se requieren; programas para preparar y facilitar a los estudiantes en el proceso de inserción en la educación superior, a través de estudios dirigidos u otras formas de afianzamiento; particularmente a los que en estos momentos estudian bajo criterios de 
Invest. pens. crit. (ISSN 1812-3864)

Vol. 7, No. 1, enero- abril 2019

pp. $05-26$

rendimiento escolar mínimo; y de manera prioritaria vincular a la academia en los diversos temas de la políticas educativas, que permitan avanzar hacia una educación de calidad

\section{Agradecimientos}

A la Universidad Católica Santa María La Antigua (USMA), ya que este es uno de los proyectos de investigación, beneficiados de las convocatorias de apoyo a la investigación científica. A todas las autoridades del Ministerio de Educación (MEDUCA) la cuál sin el acceso a los datos, no hubiera sido posible esta investigación, en especial mención a la Dra. Marisa Montesano de Talavera y a la Mgter Linda Moreno. De igual forma al IFARHU, el cual nos brindó información importante, especialmente al Mgter Anibal Barnett.

\section{Bibliografía}

\section{Libros, Revistas e Informes}

Arcia Jaramillo, O. (24 de marzo de 2017). Presupuesto: Subsidios Estatales Continúan Creciendo. La Prensa, pág. Panorama.

Denzin, N. (1970). Sociological Methods: a Source Book. Chicago: Aldine Publishing Company.

Espínola, E. Y. (2002). Educación y Conocimiento; Una nueva Mirada. La Deserción Escolar en América Latina: Un Tema Prioritario para la Agenda Regional. Revistas Iberoamericana de Educación.OEI, 30.

Gálvez Amores, I. G. (2017). Importancia del Clima Escolar en el Rendimeinto Académico en las Escuelas Panameñas. Panamá: Tesis USMA-SENACYT. Proyecto ITE-15-007.

Herrera, L. C., Torres-Lista, V., \& Montenegro, M. (2018). Analysis of the State Budget for Education of the Republic of Panama from 1990 to 2017. International Education Studies, 11(7), 71-82.

IFARHU. (2012). Memoria Institucional. Panama: IFARHU.

IFARHU. (2013). INFORME ANUAL PRESENTADO A LA ASAMABLEA NACIONAL. PANAMA: IFARHU.

IFARHU. (2016). Manual de Procedimiento de Becas Universal. Panamá: IFARHU.

Merton, R. (1964). Teoría y Estructuras Sociales. México DF: Fondo de Cultura Económica.

Noriega Martinez, G. (2017). Importancia de la Autoestima y Motivación Escolar en las Escuelas Panameñas,. Panamá: Tesis USMA-SENACYT.Proyecto ITE.15-007.

OEA, A. (2003). Estrategias y Materiales Pedagógicos para la Retención Escolar. Buenos Aires: Ministerio de Educación, Ciencia y Tecnología.

OEA, AICD. (2003). Estrategias y Materiales Pedagógicos para la Retención Escolar. Buenos Aires: Ministerio de Educación, Ciencia y Tecnología. 
Invest. pens. crit. (ISSN 1812-3864)

Vol. 7, No. 1, enero- abril 2019

pp. $05-26$

Orozco Cruz, J. C., Alfredo, O. T., \& Vivian, V. D. (2009). ¿CALIDAD DE LA EDUCACIÓN O EDUCACIÓN DE CALIDAD? UNA PREOCUPACIÓN MÁS ALLÁ DEL MERCADO. REVISTA IBEROAMERICANA DE EDUCACIÓN. , 161-181.

Paredes, M. (2015). EDUCACION BILINGUE DE CALIDAD PARA TODOS. PANAMA: MEDUCA.

Pino, Y. (10 de junio de 2012). La "beca universal" que no es tan universal. La Estrella de Panamá, pág. Nacional.

RAE. (2014). Diccionario de La Lengua Española. Madrid: Espasa.

Rivera Camacho, E. C. (2018). Resiliencia:Factores Protectores que Intervienen en la Calidad Educativa en las Escuelas Panameñas. Panamá: USMA-SENACYT. Proyecto ITE 15-007.

Sánchez, D. (26 de Septiembre de 2016). Estamos creando una mediocridad. Día a Día.

Sarmiento Goméz, A. (2006). Una Estrategia para Aumentar la Retención de los Estudiantes. Bogota: Ministerio de Educación.

Sokol, J. B. (2016). Crecimiento con Equidad e Inclusión: Politicas Públicas en la Economía anameña para lograrlo. Investigación y Pensamiento Crítico.Revista de investigación de la universidad Católica Santa María la Antigua de Panamá, 1-102.

\section{Normas, Leyes y Decretos.}

Constitución Política de la República de Panamá. Texto único 2004.

Ley 47 de 1946, Orgánica de Educación, con las modificaciones de la Ley 34 de 1995.

Ley 40 de 23 de enero de 2010, Que regula el Programa e Beca Universal, y modifica un artículo de la Ley 8 del 2010, relativo al Financiamiento del Programa”. Gaceta oficial, 26604 de 23 de agosto de 2010.

Decreto Ejecutivo No.1 De 12 de enero de 2011 "Que el reglamenta la Ley 40 de 2010, relativo al financiamiento del Programa."

Ley No.14, de 12 de agosto de 2014, que modifica y adiciona articulas a la Ley 40 de 2010 y aumenta la asignación de la Beca Universal Gaceta Oficial No.27598-B, de 12 de agosto de 2013.

Decreto Ejecutivo No.55 de 24 de febrero de 2015, que reglamenta la Ley 40 de 23 de agosto de 2010, modificada y adicionada por la Ley no.14 de 12 de agosto de 2014.

Ley 389 de 22 de marzo de 2017, que amplía beneficio a alumnos de escuelas y colegios particulares. Gaceta Oficial No.28243.

Resolución N³20-2016-735, (De miércoles 28 de septiembre de 2016) "Por el cual sé que aprueba el documento titulado "Manual de Procedimientos de Beca Universal". Gaceta Oficial No. N 28136-A del 11 de octubre de 2016. 